\title{
Feasibility of a continuous, multi-sensor remote health monitoring approach in persons living with neurodegenerative disease
}

\author{
F. Elizabeth Godkin ${ }^{1}$ - Erin Turner ${ }^{1}$ - Youness Demnati ${ }^{1}$ - Adam Vert ${ }^{1}$ - Angela Roberts ${ }^{2,3}$ - Richard H. Swartz ${ }^{4,5}$. \\ Paula M. McLaughlin ${ }^{7}$. Kyle S. Weber ${ }^{1}$. Vanessa Thai ${ }^{1}$ - Kit B. Beyer ${ }^{1}$ - Benjamin Cornish ${ }^{1}$. Agessandro Abrahao ${ }^{4,5}$. \\ Sandra E. Black ${ }^{4,5}$. Mario Masellis ${ }^{4,5} \cdot$ Lorne Zinman $^{4,5} \cdot$ Derek Beaton $^{8} \cdot$ Malcolm A. Binns $^{8,9} \cdot$ Vivian Chau $^{8}$. \\ Donna Kwan ${ }^{6} \cdot$ Andrew Lim ${ }^{4,5} \cdot$ Douglas P. Munoz $^{6} \cdot$ Stephen C. Strother ${ }^{8,10} \cdot$ Kelly M. Sunderland ${ }^{8} \cdot$ Brian Tan $^{8}$. \\ William E. Mcllroy ${ }^{1} \cdot$ Karen Van Ooteghem ${ }^{1}[$
}

Received: 10 July 2021 / Revised: 27 September 2021 / Accepted: 28 September 2021 / Published online: 27 October 2021

(c) Crown 2021

\begin{abstract}
Background Remote health monitoring with wearable sensor technology may positively impact patient self-management and clinical care. In individuals with complex health conditions, multi-sensor wear may yield meaningful information about health-related behaviors. Despite available technology, feasibility of device-wearing in daily life has received little attention in persons with physical or cognitive limitations. This mixed methods study assessed the feasibility of continuous, multisensor wear in persons with cerebrovascular (CVD) or neurodegenerative disease (NDD).

Methods Thirty-nine participants with CVD, Alzheimer's disease/amnestic mild cognitive impairment, frontotemporal dementia, Parkinson's disease, or amyotrophic lateral sclerosis (median age 68 (45-83) years, 36\% female) wore five devices (bilateral ankles and wrists, chest) continuously for a 7-day period. Adherence to device wearing was quantified by examining volume and pattern of device removal (non-wear). A thematic analysis of semi-structured de-brief interviews with participants and study partners was used to examine user acceptance.

Results Adherence to multi-sensor wear, defined as a minimum of three devices worn concurrently, was high (median $98.2 \%$ of the study period). Non-wear rates were low across all sensor locations (median 17-22 min/day), with significant differences between some locations $(p=0.006)$. Multi-sensor non-wear was higher for daytime versus nighttime wear $(p<0.001)$ and there was a small but significant increase in non-wear over the collection period $(p=0.04)$. Feedback from de-brief interviews suggested that multi-sensor wear was generally well accepted by both participants and study partners.

Conclusion A continuous, multi-sensor remote health monitoring approach is feasible in a cohort of persons with CVD or NDD.
\end{abstract}

Keywords Wearable sensors · Neurodegenerative disease $\cdot$ Cerebrovascular disease · Adherence $\cdot$ User acceptance . Remote monitoring

Karen Van Ooteghem

kvanooteghem@uwaterloo.ca

1 Department of Kinesiology and Health Sciences, University of Waterloo, Waterloo, ON, Canada

2 School of Communication Sciences and Disorders, Elborn College, Western University, London, ON, Canada

3 Roxelyn and Richard Pepper Department of Communication Sciences and Disorders, Northwestern University, Evanston, IL, USA

4 Dr. Sandra Black Centre for Brain Resilience and Recovery, Hurvitz Brain Sciences Research Program, Sunnybrook Research Institute, University of Toronto, Toronto, ON, Canada
5 Division of Neurology, Department of Medicine, Sunnybrook Health Sciences Centre, University of Toronto, Toronto, ON, Canada

6 Centre for Neuroscience Studies, Queen's University, Kingston, ON, Canada

7 Nova Scotia Health Authority, Halifax, NS, Canada

8 Rotman Research Institute, Baycrest Health Sciences, Toronto, ON, Canada

9 Dalla Lana School of Public Health, University of Toronto, Toronto, ON, Canada

10 Department of Medical Biophysics, University of Toronto, Toronto, ON, Canada 


\section{Introduction}

Advances in wearable sensor technology have made it possible to monitor aspects of health outside of the clinic, capturing data that can positively impact clinical care, patient self-management of symptoms, and overall health [1]. For persons with complex health conditions such as those living with neurodegenerative disease (NDD) or cerebrovascular disease (CVD), remote monitoring, supplementary to clinical care, has the potential to improve quality of life and health outcomes by improving disease tracking, monitoring treatment adherence and effectiveness, and improving healthcare decision-making [2-5]. Objective monitoring using remote technologies may also reduce reliance on selfreport measures and barriers to healthcare access for those living in remote regions or those who live long distances from the clinics that provide specialized care [6-8]. Remote monitoring is particularly valuable for recording behaviors that occur sporadically and thus may not manifest during clinical appointments, behaviors that vary over time, and those symptoms that present differently in clinic versus at home $[4,9,10]$. Wearable technology, when paired with telemedicine as a virtual care tool, has also demonstrated clinical utility in the context of the COVID-19 pandemic [11-13]. Further, extended sensor wear in the home environment can reveal patterns in health-linked behaviors that can be captured with greater ecological validity and fidelity than point-in-time assessments or symptom diaries $[14,15]$.

The focus of the current work, conducted as part of the Ontario Neurodegenerative Disease Research Initiative (ONDRI), is directed to the application of wearable sensors for those with NDD and CVD; however, the implications of the work extend to many other clinical populations. To maximize the clinical utility of wearable sensors, they must be acceptable to end-users and capable of capturing data relevant to behaviors and outcomes that are important to clinical decision-making. Thus, it is important to consider inputs from clinicians, patients, and care partners regarding their needs and concerns related to wearable sensors. Moreover, sensor systems must be built with enough flexibility to suit a variety of potential applications, including tailored use to meet specific needs $[16,17]$. These matters are becoming increasingly important as evidence accumulates in support of assessing multiple domains of health (e.g., sleep, activity, social engagement, cognition), independently or with respect to one another, to account for the multiple impairments that occur in complex health conditions [6]. For example, continuous, 24-h monitoring with wearable sensors presents an opportunity to objectively quantify both activity and sleep to understand circadian rest-activity rhythms [18], and the relationship between physical activity, sedentary behavior, and sleep [19,20].
To evaluate multiple domains in a unified way, biometric signals must be captured from different sensor types (multimodal) embedded in one or more devices, often worn on different body segments (multi-nodal). Combining signals from different modes and nodes can also reduce uncertainty associated with quantifying free-living data, by leveraging complementary sources of information to characterize behavior. This is especially useful when target measures cannot be captured directly (e.g., sleep quality, social engagement). A multi-sensor approach must address the burden that accompanies multiple modes and nodes by optimizing user experience and reducing the number of sensors needed to the minimum necessary for the measures of interest; a balance that has been referred to as the benefit-to-burden ratio [17]. Importantly, this does not mean that a single-device solution (e.g., one wrist worn device) is the best option, since this approach can compromise data quality and clinical utility. For example, in the assessment of walking, a single device is limited in its ability to accurately detect gait parameters used to describe left-right asymmetry, compared to devices worn bilaterally at the ankle [21]. In Parkinson's disease, recent evidence supports the use of multiple sensors for motor symptom detection outside of highly controlled settings, such as the occurrence of freezing of gait [4, 22]. Also, in both the activity and sleep domains, adding heart rate and/or skin temperature to accelerometry has been shown to improve the accuracy of outcomes [23, 24].

Despite significant advances in wearable sensor technology, there remains a relative lack of studies formally examining the feasibility of continuous monitoring of free-living behavior in persons with physical or cognitive limitations that could uniquely impact acceptance and adherence (i.e. willingness to wear and commitment to prescribed wear schedule) $[25,26]$. The literature tends to focus on issues of technical efficacy such as precision and reliability [27] and while warranted, there remains need to understand user acceptance and adherence to device-wearing in daily life. Among studies that have evaluated feasibility from this perspective in those with impaired function or cognition, acceptability is generally high [26, 28-30], with reduced adherence linked to high user engagement requirements [31], multi-day wear [26], and specific cohorts such as those living with dementia [32]. Most studies, however, have focused on a single device and daytime wear [27,32], with specific gaps surrounding the feasibility of extended duration, multi-sensor wear paradigms.

The goal of this study was to examine the feasibility of a multi-sensor approach used to capture activity, mobility, sleep, and cardiovascular function continuously over seven days in persons living with CVD or a range of NDDs. Specifically, the study investigated the following: (1) adherence to device-wearing based on sensor location, (2) adherence to multi-sensor device wearing based on time of day and wear duration, and (3) participant and study partner acceptance of continuous multi-sensor wear for a week-long period. 
Adherence was evaluated by examining volume and pattern of device removal, which were derived from sensor data, while acceptance was examined through de-brief interviews with participants and study partners. Study partners were included given the important role that care partners play in the daily life of the patient and their potential involvement in technology implementation [33]. We hypothesized that adherence to and acceptance of a multi-sensor set-up would be high, as demonstrated by low device non-wear time and positive participant and study partner reports of their experience. We also hypothesized that differences in adherence would emerge based on disease cohort, sensor type or wear location, time of day, and wear duration.

\section{Methods}

\section{Participants}

The ONDRI Remote Monitoring in Neurodegenerative Disease (ReMiNDD) study aimed to recruit 50 participants (minimum 5 per cohort from CVD, Alzheimer's disease/ amnestic mild cognitive impairment (AD/MCI), frontotemporal dementia (FTD), Parkinson's disease (PD), and amyotrophic lateral sclerosis (ALS)) from tertiary care clinics at Sunnybrook Health Sciences Centre in Toronto, Canada. In addition to meeting standard clinical diagnostic criteria, as confirmed by a neurologist and detailed previously in Farhan et al. [34], inclusion criteria included the following: (1) a self-reported level of proficiency in speaking and understanding English as determined by the Language Experience and Proficiency Questionnaire (LEAP-Q) [35] and (2) having a study partner known to the participant for at least 2 years, who could respond to care partner questionnaires and attend both a baseline and discharge visit. Participants were not eligible if they experienced contraindications as outlined by device manufacturers or an allergy/sensitivity to the materials used to mount the devices. Following prescreening by a clinical study coordinator, physicians verified eligibility and either introduced the study to patients during a clinic visit or via telephone or gave permission to a ReMiNDD research coordinator to do the same. At the time of consent, participants were informed they would receive a personalized feedback report based on their sensor data. This study was approved by the Sunnybrook Health Sciences Centre research ethics board and all participants provided written informed consent prior to data collection.

\section{Procedures}

The study was completed between May 2019 and March 2020. Data collection consisted of a baseline clinic visit, a 7-day free-living sensor wear period, and a discharge visit either in clinic or at home, as preferred by the participant. During the baseline visit, participants and study partners completed a detailed health history and several clinical assessments. Participants were also instrumented with five wearable devices that they were oriented to with information about what they measured and instructions for donning/ doffing. Participants were asked to wear limb devices bilaterally on wrists and ankles, as well as a chest device, $24 \mathrm{~h}$ per day, except for bathing or swimming. All devices were data logging. Device instruction cards and paper logs to record notable events (e.g., device removal, bed/wake times) were provided as part of a study kit. Logs were used by the study team, when necessary, to cross reference against sensor data. Participants were encouraged to go about their usual activities during the free-living wear period. Approximately 2 days after the baseline visit, a member of the study team completed a telephone check in with participants to answer any questions regarding sensor wear. During the discharge visit, devices were returned and a study de-brief interview was conducted separately with participants and study partners (where possible).

\section{Data collection}

\section{Demographic and clinical measures}

Demographic and health history information, as well as the following clinical assessments, were collected either by selfreport or by a trained member of the study team as part of the ONDRI ReMiNDD study: Pittsburgh Sleep Quality Index (PSQI) [36], General Anxiety Disorder-7 (GAD-7) [37], Quick Inventory of Depressive Symptomology-SelfReport (QIDS-SR) [38], Montreal Cognitive Assessment (MoCA) [39] and the modified Rankin Scale (mRS) [40]. This information was collected and stored using REDCap electronic case report forms (www.project-redcap.org) and hosted on Brain-CODE (www.braincode.ca) [41, 42]. The broader ReMiNDD study also included additional clinical scales, a detailed neuropsychological assessment, blood work, one night of pulse oximetry, and an optional clinical MRI.

\section{Wearables data collection}

Limb-worn devices were GENEActiv Originals (43 mm $\times 40 \mathrm{~mm} \times 13 \mathrm{~mm})($ ActivInsights, UK), each with a triaxial accelerometer as well as a temperature and light sensor (Fig. 1a). These were fastened using rubber watch straps (wrists) or Fabrifoam (Fabrifoam, PA) medical-grade wraps (ankles). The ankle devices were positioned just above the medial or lateral malleolus, based on participant preference (Fig. 1b). Accelerometer and temperature data were collected at a sampling frequency of $75 \mathrm{~Hz}$ and $0.25 \mathrm{~Hz}$, 


\begin{tabular}{|c|c|c|}
\hline Device & Sensors & Location(s) \\
\hline & $\begin{array}{c}\text { Tri-axial accelerometer } \\
\text { Near-body temperature } \\
\text { Light }\end{array}$ & Bilateral wrists \\
\hline & $\begin{array}{c}\text { Tri-axial accelerometer } \\
\text { Near-body temperature } \\
\text { Light }\end{array}$ & Bilateral ankles \\
\hline (6) & $\begin{array}{l}\text { Electrocardiography } \\
\text { Tri-axial accelerometer }\end{array}$ & $\begin{array}{l}\text { Chest } \\
\text { (vertical or horizontal } \\
\text { orientation) }\end{array}$ \\
\hline
\end{tabular}

Fig. 1 Multi-modal, multi-nodal device-sensor relationship and participant set up. a Limb (GENEActiv) and chest (Bittium Faros 180) devices worn within this study, with the corresponding sensors and body location(s) and $\mathbf{b}$ Schematic representation of device location

respectively. The chest-worn device was a Bittium Faros $180(48 \mathrm{~mm} \times 29 \mathrm{~mm} \times 12 \mathrm{~mm})$ (Bittium, Finland), which included electrocardiography (ECG) and a tri-axial accelerometer. The chest device was limited to collecting data for the first 4 days of the study period, due to battery life. The decision to restrict collection to a single charge for the chest device was motivated by a desire to minimize participant burden associated with transferring data and device charging. The chest device was mounted using a 2-lead FastFix electrode adhesive as per the manufacturer's recommendation (Fig. 1b). Participants with chest hair were asked to shave the area prior to sensor attachment. ECG data were sampled at $250 \mathrm{~Hz}$, with the built-in accelerometer sampling at $25 \mathrm{~Hz}$. The electrode adhesive was waterproof and could be left on during bathing or swimming. Participants were given additional adhesives to use as necessary during the study period.

Sensor data were purposefully oversampled with five devices to address the study team's work to develop guidelines for a minimal sensor set in NDD, which considers the need to characterize disease-specific features and subtle changes in symptoms or behavior. Specifically, the interest in capturing data bilaterally and across upper and lower limbs was driven by an understanding that persons living with CVD and NDD experience asymmetric and body segmentspecific presentation of symptoms and impairment (e.g., gait in PD, upper versus lower motor neuron degeneration in ALS) $[43,44]$. The decision to include a chest-mounted

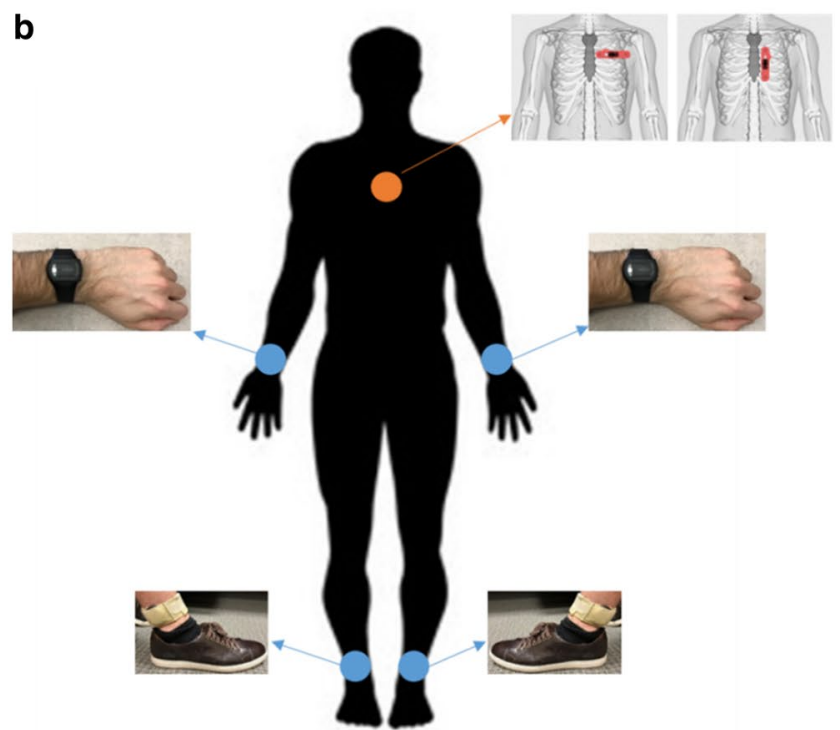

on body segments. The multi-modal, multi-nodal approach enables simultaneous assessment of multiple domains of health in persons living with cerebrovascular and neurodegenerative disease

device with both ECG and accelerometry capabilities was guided by evidence to suggest that continuous ECG outperforms proxy measures obtained via photoplethysmography (PPG) [45]. There was also an interest in examining autonomic function and other relevant measures derived from a sensor located on the chest, such as speech or respiration, which are known to be affected in persons with CVD and NDD [46-48].

\section{De-brief interviews}

A qualitative descriptive approach was used as a framework for the collection and analysis of data [49], as has been used previously when gathering perspectives on technology in clinical research [50]. Interviews were semi-structured and lasted approximately $10 \mathrm{~min}$. Interviews were conducted by two research coordinators (ET, YD) who worked with the participants and study partners throughout the study. An interview guide consisting of open-ended questions and probes related to user experience with the sensors was used to conduct the interview. Interviewers explored specific issues raised by the participant or study partner in greater depth, where relevant. With permission, all interviews were digitally recorded for offline analysis. 


\section{Data processing and analysis}

All data, except the de-brief interview recordings, were subscribed to ONDRI's data curation processes, which were developed to optimize quality assurance and quality control procedures for large, complex datasets [51].

\section{Sensor non-wear}

At the end of the data collection period, devices were returned to the study team where raw data were offloaded from each device for processing. Data files were visually inspected to identify periods when the devices were not worn (i.e. non-wear). Visual inspection was used to achieve temporal resolution not currently afforded by published nonwear algorithms, which typically focus on periods with a minimum threshold of 30-60 min [52]. Non-wear detection criteria included the absence of acceleration and a sustained decrease in temperature for a minimum of 5 minutes. Non-wear was expressed as a proportion of a standardized amount of wear time, either four consecutive 24-h periods of wear ( $2 \mathrm{pm}$ to $2 \mathrm{pm}$ ) to examine the effect of sensor location (included all devices) or six consecutive 24-h periods of wear to examine the effect of time of day and number of days worn (included limb devices only).

Given the desire to understand acceptance and adherence to a multi-sensor, extended wear model, a subcomponent of the analysis focused on the six 24-h periods obtained from the limb-worn devices. Specifically, by identifying periods of time when a minimum of three limb-devices were worn, adherence was assessed for a sensor set that captures data bilaterally (i.e. both ankles or both wrists) and provides representation from both the upper and lower body (i.e. minimum of 1 wrist and 1 ankle). Instances when this multi-sensor criterion were not met were then termed "multi-sensor non-wear". Non-wear data were examined for both volume and pattern at the group and participant level over the course of the collection period. For participants who exhibited the greatest non-wear rates (outliers defined as participants with a proportion of non-wear greater than 1.5 times the interquartile range from the third quartile), demographic and clinical data were inspected to explore whether adherence to the study protocol may have been impacted by clinical characteristics.

\section{De-brief interviews—user experience}

Digital recordings of participant and study partner interviews were transcribed by a research team member (VT). A qualitative content analysis [49] was performed by two members of the research team (ET, VT) who read and opencoded the transcripts before meeting to develop a coding framework, which they reviewed with the research team prior to organizing the data into themes. Throughout data analysis, ET, VT, FEG, and KVO discussed and refined the themes until agreement was reached. Thematic summaries considered all participants and were not divided by disease cohorts due to relatively small numbers within each group.

\section{Statistical analyses}

Demographic and clinical data were summarized using descriptive statistics. Non-wear data were non-normally distributed as determined using the Shapiro-Wilk test and as such, were analyzed using non-parametric tests. A Friedman test was used to compare average non-wear rates between wear locations. The Wilcoxon matched pairs signed rank test was used to compare multi-sensor non-wear rates for day (7:00am-11:00pm) versus night (11:00pm-7:00am). A nonparametric longitudinal data analysis [53] was used to examine multi-sensor non-wear rates across the wear duration. Post-hoc tests were conducted using the Nemenyi test. Given the small number of participants within disease cohorts, the data were not statistically analyzed for between-cohort differences. Statistical analyses were conducted in SPSS (IBM SPSS Statistics for Windows, Version 27.0. Armonk, NY: IBM Corp). Statistical significance was set at $\alpha<0.05$ and all data are presented as medians (range).

\section{Results}

\section{Study overview}

A total of 123 patients living with CVD, AD/MCI, FTD, PD, or ALS were contacted. Of these, 44 agreed to participate and another six agreed to review the study information letter. Due to the COVID-19 pandemic and the need to terminate recruitment efforts based on local restrictions, 39 participants completed the study. The primary reason for declining participation was general research study fatigue, which may have been related to the study site that afforded many, ongoing opportunities for study participation.

\section{Participant characteristics}

Participants were sampled from the five cohorts as follows: $10 \mathrm{CVD}, 8 \mathrm{AD} / \mathrm{MCI}, 5 \mathrm{FTD}, 11 \mathrm{PD}$, and 5 ALS. The median age was $68(45-83)$ years and $14(36 \%)$ participants were females. Table 1 summarizes demographic and clinical characteristics of participants, presented overall and for each of the five disease cohorts. The study partner for most participants $(n=36,92 \%)$ was a spouse or partner, while three participants were enrolled with a friend or sibling (median 
Table 1 Participant characteristics

\begin{tabular}{|c|c|c|c|c|c|c|}
\hline & Overall & $\operatorname{CVD}(n=10)$ & $\mathrm{AD} / \mathrm{MCI}(n=8)$ & $\operatorname{FTD}(n=5)$ & $\mathrm{PD}(n=11)$ & $\operatorname{ALS}(n=5)$ \\
\hline Age (years) & $68(45-83)$ & $72(55-76)$ & $73(62-82)$ & $57(48-83)$ & $65(59-76)$ & $65(45-73)$ \\
\hline Sex $(n, \%$ Female $)$ & $14(36 \%)$ & $3(30 \%)$ & $2(25 \%)$ & $2(40 \%)$ & $5(45 \%)$ & $2(40 \%)$ \\
\hline $\begin{array}{l}\text { Education }(n, \% \text { with post- } \\
\text { secondary education) }\end{array}$ & $35(90 \%)$ & $9(90 \%)$ & $8(100 \%)$ & $5(100 \%)$ & $10(91 \%)$ & $3(60 \%)$ \\
\hline $\operatorname{MoCA}(0-30)^{\mathrm{a}}[n=32]$ & $25(16-30)$ & $27(16-30)$ & $24(22-27)^{\mathrm{e}}$ & $\ddagger \llbracket$ & $27(17-28)^{\mathrm{d}}$ & $24(22-25)^{d}$ \\
\hline $\operatorname{mRS}(0-5)^{\mathrm{b}}[n=33]$ & $2(0-4)$ & $1(0-3)^{\mathrm{d}}$ & $2(0-3)$ & $3(3-4)^{\mathrm{d}}$ & $2(1-3)^{\mathrm{e}}$ & $\ddagger$ \\
\hline PSQI $(0-21)^{\mathrm{b}}[n=33]$ & $6(0-14)$ & $5(0-9)^{\mathrm{e}}$ & $4(1-7)^{\mathrm{d}}$ & $11(5-12)^{\mathrm{d}}$ & $5(2-14)^{\mathrm{e}}$ & $8(4-13)$ \\
\hline GAD-7 $(0-21)^{\mathrm{b}}[n=38]$ & $2(0-16)$ & $1(0-12)$ & $1(0-4)$ & $2(0-7)^{\mathrm{d}}$ & $4(0-9)$ & $5(0-16)$ \\
\hline QIDS-SR $(0-26)^{\mathrm{bc}}[n=37]$ & $5(1-12)$ & $3(2-10)$ & $3(1-8)^{\mathrm{d}}$ & $4(2-12)^{\mathrm{d}}$ & $8(3-12)$ & $9(6-12)$ \\
\hline
\end{tabular}

MoCA Montreal Cognitive Assessment, $m R S$ Modified Rankin Scale, PSQI Pittsburgh Sleep Quality Index, GAD-7 General Anxiety Disorder-7, $Q I D S$-SR Quick Inventory of Depressive Symptomology-Self-Report

Data are presented as median (range) unless otherwise noted

${ }^{a}$ Higher score indicates greater functioning

${ }^{\mathrm{b}}$ Lower score indicates greater functioning

${ }^{\mathrm{c}}$ Total score for QIDS-SR adjusted to 26 (question 12 was not collected as a part of the ReMiNDD study)

Missing data for: ${ }^{\mathrm{d}}$ one participant; ${ }^{\mathrm{e}}$ Two participants

${ }^{\ddagger}$ For subgroups of less than four participants ( 3 or fewer scores), cohort level summaries are not reported

${ }^{\mathbb{I}}$ Missing MoCA scores were attributed to participants who were unable to complete the assessment due to cognitive impairment

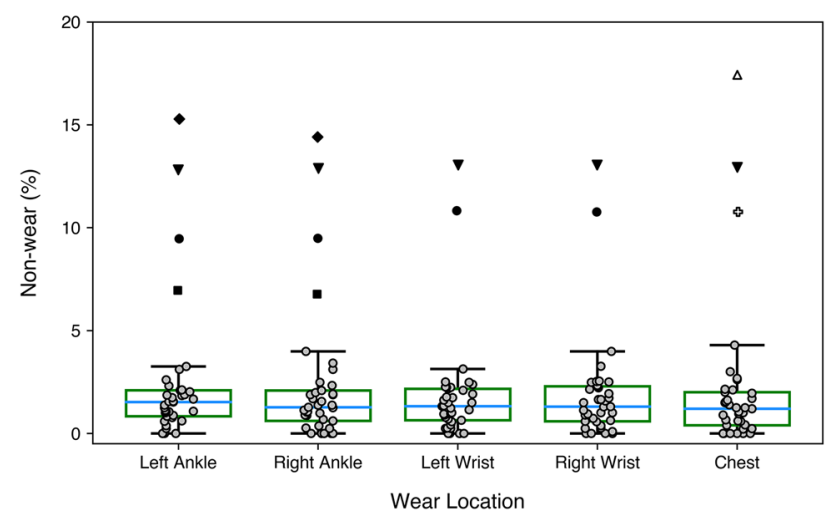

Fig. 2 Participants' average non-wear rate by wear location $(n=37)$. The data collection window was defined as $2: 00 \mathrm{pm}$ on the day of the baseline visit to 2:00 pm on Day 4, resulting in four consecutive 24-h periods. Grey symbols denote individual participants. Black symbols denote the four participants deemed to be outliers across two or more wear locations. White symbols denote participant outliers for a single wear location. Non-wear rate was significantly different dependent upon wear location $(p=0.006)$ and post-hoc testing revealed a significant difference between the chest compared to the left wrist $(p=0.03)$ and right wrist $(p=0.02)$. Note: symbols appearing across figures do not denote the same participant

age was 65 (41-80) years and 27 (69\%) study partners were females). Previous work within ONDRI showed similar group composition when a study partner was required [54]. All participants completed the baseline clinic visit, at-home monitoring period, and discharge visit; however, not all participant/study partner pairs completed all clinical scales or the de-brief interviews.

\section{Non-wear by location}

The full device set was worn for the first 4 days, after which time the chest device battery was deplete and the device was removed. All four limb devices continued to be worn for the remaining 2 days. Given our interest in potential differences in adherence between the limb and chest devices, the effect of wear location was examined for this 4-day period. Median non-wear rates ranged from 1.2 to $1.5 \%$ per day (17-22 $\mathrm{min} /$ day) across all wear locations (Fig. 2). Non-wear rate was significantly different dependent upon wear location $\left(\chi_{(4)}^{2}=14.355, p=0.006\right)$ Post-hoc testing revealed a significant difference between the chest compared to the left wrist $(p=0.03)$ and right wrist $(p=0.02)$, with greater non-wear rates occurring for both wrists versus the chest. Four participants had non-wear rates that were classified as outliers (exceeded the upper quartile by 1.5 times the interquartile range) for more than one wear location (e.g., both ankles), but only one participant had non-wear rates classified as outliers for all wear locations. Data from one participant were omitted due to a device issue and another due to an unrelated ankle issue that prevented the device from being worn. Analysis was based on data from 37 participants. 


\section{Multi-sensor non-wear}

As noted, evaluation of non-wear based on time of day, wear duration, and between individual study participants considered multi-sensor non-wear to be any time when less than three limb devices were worn (i.e. two or more limb devices were removed). Multi-sensor non-wear analyses were based on data from 37 participants (as described above).

\section{Group level non-wear}

Multi-sensor non-wear, as defined, ranged from 0 to $30.3 \%$ of the study period. Within each cohort, multi-sensor nonwear occurred for a median (range) of $2.3 \%$ of time in $\mathrm{AD} / \mathrm{MCI}(0-11.4 \%)$ and CVD $(0.3-8.1 \%), 2.0 \%$ of time in FTD $(0-2.2 \%), 1.5 \%$ of time in PD $(0-30.3 \%)$, and $0.9 \%$ of time in ALS (0.5-18.6\%). Overall, participants wore a minimum of three devices for a median of 5 days and $21 \mathrm{~h}$ $(98.2 \%)$ of the six 24 -h periods included in the analysis.

Multi-sensor non-wear was significantly different based on time of day $(\mathrm{Z}=-3.394, p<0.001)$, with non-wear occurring more often during the day (median $=2.4 \%$, range $=0-28.9 \%)$ than at night (median $=0 \%$, range $=0-32.9 \%$ ) (Fig. 3a). Multi-sensor non-wear rates increased over the course of the wear period $(\mathrm{T}=1.769$, $p=0.04$ ) (Fig. 3b; median non-wear rates of 0, 22, 12, 15, 16 , and 24 min from first to sixth day).

\section{Individual level non-wear}

Each participant's non-wear volume and pattern are illustrated in Fig. 4. Thirty-one participants (84\%) adhered to multi-sensor wear for more than $95 \%$ of the study period. According to self-reported device removal $\operatorname{logs}(n=29)$, bathing was the most common reason for device removal (as instructed) and only two participants noted the removal of devices due to discomfort. The five participants with the greatest non-wear rates were identified for further examination based on the outlier criterion (equated to total non-wear greater than 5\%). One of these participants (ALS5) presented with moderately severe disability (mRS score of 4), chose not to complete the cognitive assessment (MoCA), and noted concern about wearing the devices in public, specifically due to challenges with communication and a desire to avoid having to explain the sensor wearing to others. Among the remaining four participants whose non-wear was identified as an outlier, MoCA scores ranged from 24 to 28 , mRS scores were between 0 and 2, PSQI scores ranged from 0 to 7, GAD-7 scores ranged from 2 to 5, and QIDS-SR scores ranged from 2 to 8. As seen in Fig. 4, these four participants accumulated non-wear predominantly in a single, long bout
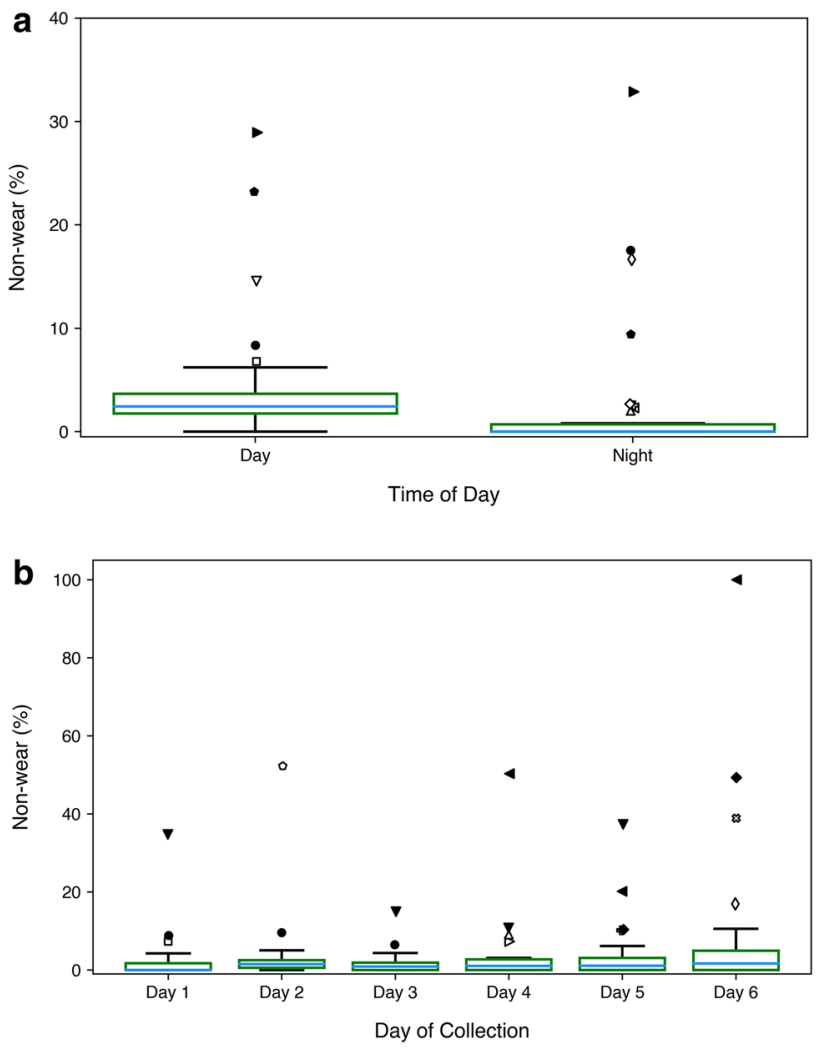

Fig. 3 Non-wear rate based on time of day (a) and day of collection (b) $(n=37)$. Data represent periods of time when less than three devices were worn (multi-sensor non-wear). The data collection window in these analyses was defined as 2:00 pm on the day of the baseline visit to 2:00 pm on Day 6, resulting in six consecutive 24-h periods. Black symbols denote the participants deemed to be outliers across two or more data points. White symbols denote participant outliers for a single data point. Non-wear rate was significantly different based on time of day $(p<0.001)$ and increased across the wear period $(p=0.04)$

(one early in collection period, three later in the collection period), whereas the participant who presented with a more distinct clinical profile (ALS5) accrued non-wear in several bouts of a moderate length. Reasons for higher non-wear values noted in these participants' logs included longer periods of time spent bathing, unintended extended removal (e.g., forgot to put back on) and life interruptions. There was no explanation provided for the extended removal in the participant presenting with the highest amount ( 30\%) of multi-sensor non-wear (PD6).

\section{De-brief interviews}

De-brief interviews were conducted with 35 participants and 27 study partners. Twenty-five of these interviews were participant/study partner pairs with interviews conducted separately. Two participant/study partner pairs declined an interview (one participant with communication difficulties, 
a

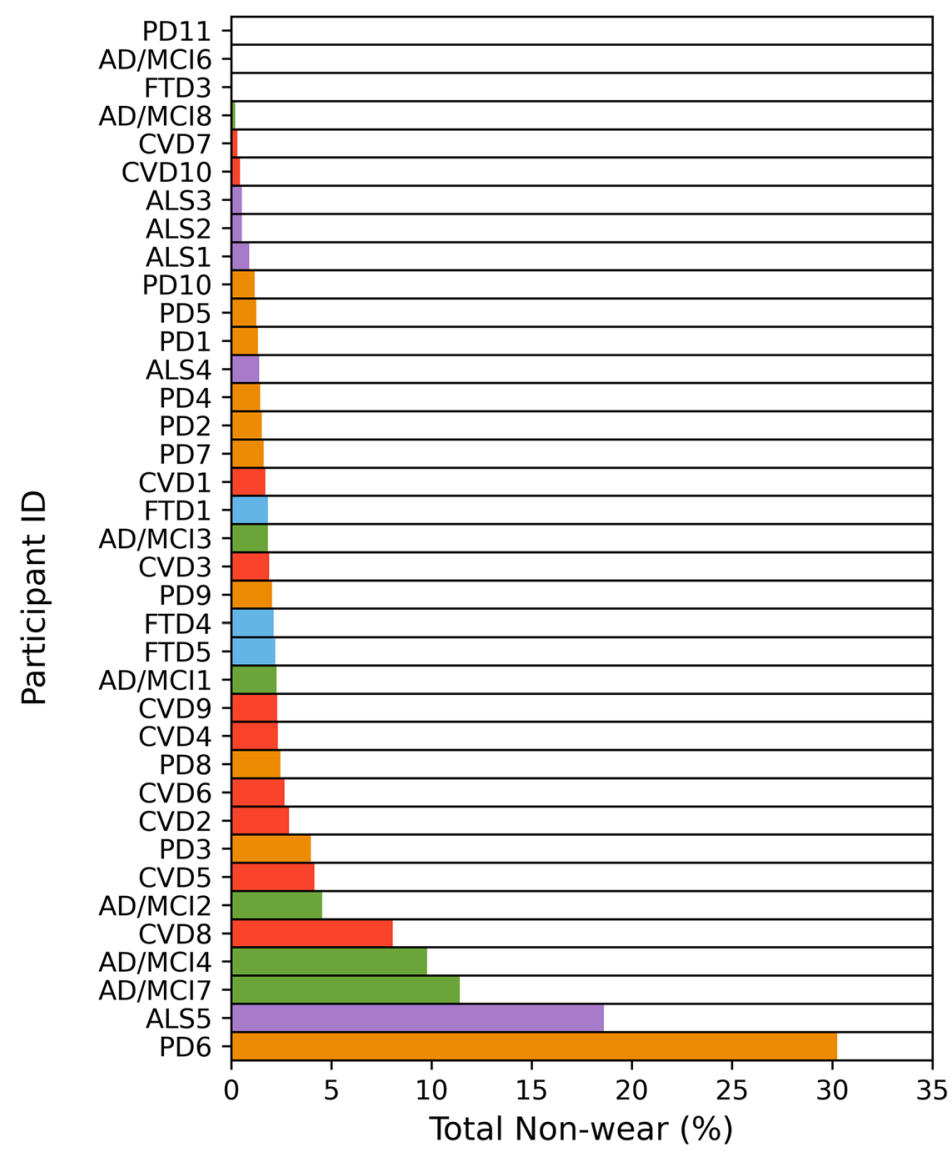

b

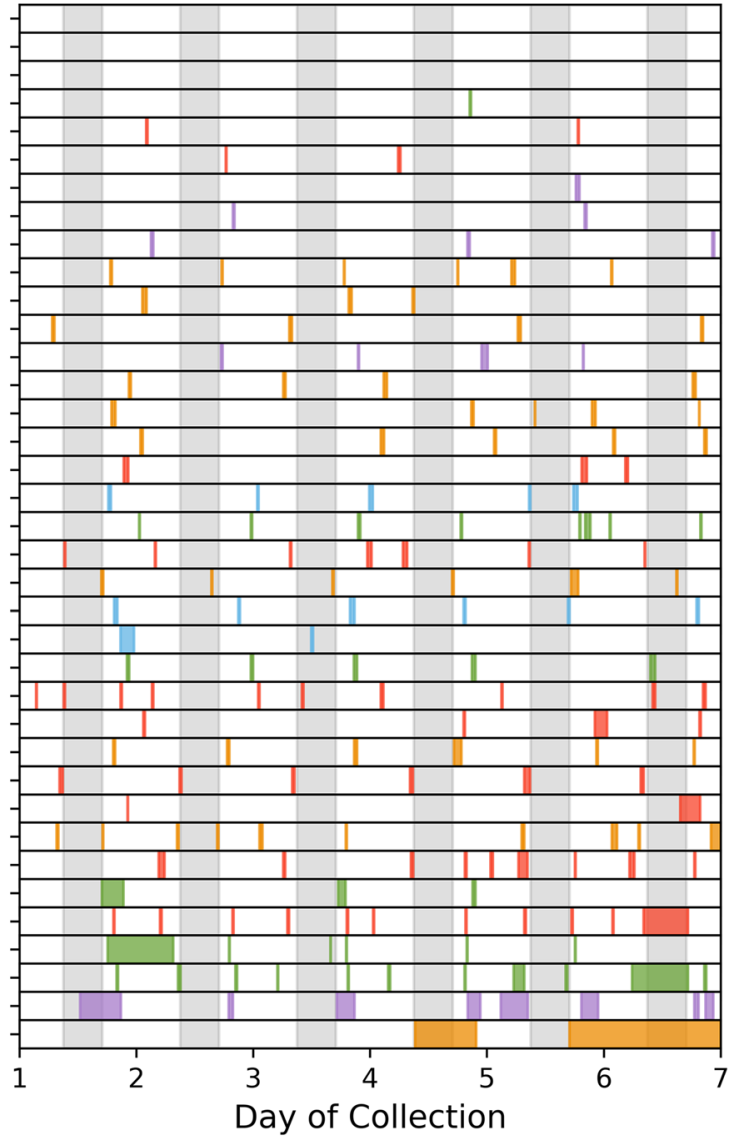

CVD

PD

ALS

FTD

$\mathrm{AD} / \mathrm{MCl}$

Fig. 4 Pattern and total rate of non-wear across and within individual study participants $(n=37)$. Data represent periods of time when less than three devices were worn (multi-sensor non-wear). a Total nonwear rates for each participant, sorted from least (top) to most (bot-

one participant with study fatigue), ten interviews were conducted with participants only, and two interviews were conducted with study partners only. In the cases where one in the pair did not interview, the reason was either that they were unavailable (one study partner) or they agreed with responses provided by their partner (nine study partners, two participants).

\section{Participant perspectives}

Overall, participants reported no considerable problems wearing multiple devices for the study duration. Qualitative analysis of the de-brief interviews resulted in four themes related to user experience with the sensors and acceptability of the device wearing protocol: comfort, ease of use, the degree of interference with activities of daily living (ADLs), tom) and $\mathbf{b}$ Non-wear periods for each day of data collection. Width of the bar denotes bout duration. Shaded grey sections denote nighttime periods (11:00 pm to 7:00am)

and appearance. For a summary of findings with representative quotes, see Table 2.

Most participants identified comfort as the key consideration related to device acceptance. Several participants distinguished between the materials that were used to affix the wrist versus ankle devices, with some noting a preference for the feeling of the medical-grade wrap that was used on the ankles. Some participants commented that the ECG adhesive was itchy or that they experienced discomfort, particularly upon removal. Comments related to ease of use were also common. Several participants noted that the devices would "move around", with a few expressing that they were uncertain about the appropriate level of tightness for the ankle strapping and therefore, that the easily adjustable wrist strap was ideal. Conversely, one participant with significant atrophy of the forearm (ALS cohort) found that the watch strap could not be tightened enough and noted an interest in using 
Table 2 Summary of findings from qualitative thematic analysis of de-brief interviews with participants and study partners

Summary Representative quotes

Comfort was deemed the most critical factor for participants, inclusive of material softness and suitability for sensitive skin

PD3: Comfort is key, especially because I wore it in such hot weather $\mathrm{AD} / \mathrm{MCI} 3$ : The material for the ankle wrappings was actually quite good. I think it was a comfortable material...So I liked that material of the softer [ankle] one better than the harder [wrist] one...

PD8: Just the sticky part [of the chest device] can become a little bit, sort of, aggravated like on your skin... I wouldn't like to keep it [on] any longer than I did because it makes your skin feel a little bit like itchy

Participants expressed the need for sensors to be easy to use, with concern about devices moving around and a need for a design permissive of independent use

CVD10: Comfort [and] ease of taking off and putting on [are important]. The look of the thing is not tremendously important to me AD/MCI8: The heart sensor, that fell off the first time. I had to push down with force to make sure it stays put

PD5: [The wrist device] wasn't too tight, not too loose. It was adjustable...The ankle sensors... sometimes they got too tight, sometimes they felt too loose

AD/MCI3: I was a little worried. It moved a lot. If I put it too tight, then it really bothered me

ALS2: I didn't do it myself [taking off and putting on devices]. My hands don't work therefore somebody else has to take them off. That's why I think the elastics would be better [for the wrist devices]

Participants noted that devices interfered with certain activities of

AD/MCI1: I have no issues. Other than if you're putting clothes on, it daily living, with some participants expressing concern about getting the sensors wet

may be more difficult. But it's a minor issue

CVD6: The ankle sensors got tangled in the sheets when I moved my ankles...they were a nuisance when that occurred

FTD4: The ones for the wrist, I found that I was getting concerned about the amount of water I might be [exposing the sensors to when] washing my hands

Participants would have preferred smaller, slimmer devices that

Study Partner: I wish it was thinner though. Thinner and it was somelooked more appealing but found the devices acceptable in this study context

thing that almost stuck to the body. Almost like a little tape thing

FTD1: The ankle ones were a little bulky. I think on the wrist, try to make it as light and small as possible

AD/MCI3: I did not mind that people see it. I just told them I was doing a study...

CVD4: The only thing they saw were my wrists and they just thought it was a wrist watch or something. Well for the ankle monitors I wore pants because I didn't want people to ask

the ankle wrap for the wrist. Two participants, who had difficulties with upper limb motor control, required assistance to attach and remove the devices and commented on the importance of a design that would enable independent use.

Although most participants did not suggest that the device set impeded their ability to perform ADLs, a few noted devices catching on clothing during dressing or on the bed sheets during sleep. Based on the instruction to remove devices for bathing or swimming, some were concerned about the sensors contacting water during ADLs such as washing dishes. Many participants commented on device size or appearance and a few mentioned that they avoided certain situations while wearing the devices (e.g., avoided wearing shorts even though it was hot) or attempted to conceal them on at least one occasion (e.g., wedding). Four participants used the word "bulky" to describe the devices and most participants who commented on size also explicitly stated a desire for smaller and slimmer devices. Despite these comments, most participants reported that the sensor set was not obtrusive, with two participants stating they "did not mind" if others saw that they were wearing devices as part of a study.

Beyond the four central themes related to user experience that emerged from the interviews, participants also made broader comments about the remote monitoring approach. A few participants expressed reluctance to wear the devices for markedly longer periods of time than the one-week study period (e.g., 6 months). Two participants explicitly stated that their willingness to wear the devices for a week stemmed from a belief that they would be able to provide valuable information to their clinician that could benefit their health and the health of people living with their disease. 


\section{Study partner perspectives}

Generally, study partners expressed that they did not have any concerns with devices used in this study. Several study partners commented on the practical aspects of devicewearing, including a desire for devices to be thinner to make them more manageable in daily life (Table 2 ). Some study partners also commented on the potential utility of receiving real-time feedback or summary results from the sensor data, with three explicitly stating an interest in learning more about disease-related outcomes such as medication impact or fall risk and health-related behaviors such as sleep quality.

\section{Discussion}

The findings of this study demonstrate feasibility of a continuous multi-sensor approach to remote monitoring for persons living with CVD and NDD, with high adherence during both day and nighttime wear across the study period. Specifically, $84 \%$ of participants wore at least three limb devices for more than $95 \%$ of the study period. Further, participants described their experience to be generally positive with respect to comfort, ease of use, and integration of the wearables into daily life. Support for the deployment of a multi-sensor approach within CVD and multiple NDD cohorts has important implications for advancing the use of remote health monitoring in persons living with complex health conditions. In such cases, there may be value in examining multiple symptoms and behaviors to understand health status and to support selfmanagement and clinical care.

Previous work examining acceptance and adherence to device wearing in daily life in NDD populations has occurred most commonly in PD [26, 30, 31] or AD [32], with a paucity of studies incorporating other NDD cohorts [28]. Furthermore, few studies have evaluated acceptability and adherence to multiple sensors worn on various body segments $[28,30,55]$, with some studies using the term "multi-sensor" to describe a single sensor plus smartphone for participant data entry [29, 31, 56]. Adams et al. [28] did examine a multi-modal, multi-nodal wear model by surveying persons with PD, Huntington's disease (HD), and prodromal HD. Most participants (86\%) reported that they were "willing or very willing" to wear the sensor set again; however, wear time was relatively short at 2 days [28]. The high wear time across all cohorts within the present study supports the general feasibility of a multi-sensor approach over an extended period and provides specific evidence from cohorts that have been understudied to date, including ALS and FTD. Additionally, high wear rates across the five wear locations (median non-wear 17-22 min/day) demonstrate the potential for system "flexibility", such that different combinations and placements of sensors may be used, with comparable acceptance and adherence, to optimize data capture for a specific purpose, including the detection of subtle changes in symptoms or behavior over time.

Overall, multi-sensor adherence was high throughout the study period, with total non-wear time largely influenced by a few participants who removed devices for hours at a time (e.g., PD6). Group level analyses revealed that multisensor non-wear rates were higher in the day versus night (median non-wear 2.4 versus $0 \%$ ), although this can be at least partially accounted for by the need to remove devices when bathing/swimming as instructed. A high nighttime adherence rate adds to previous literature that did not assess nighttime wear $[26,55,56]$ and contrasts reports of poorer nighttime compliance or refusal to wear a wrist-based sensor at night, particularly in people with dementia [32]. The difference in findings related to nighttime adherence may be attributed to the fact that some previous work has focused on participants who report regular sleep disturbances and involved protocols requiring daily donning and doffing [57], which may make overnight wear less achievable. High 24-h wear rates across a 6-day window is notable in the context of the minimum number of hours needed to report on daily behaviors (e.g., 10-h wake time per day for activity) [58] and evidence that a week-long wear period, inclusive of sleep, is important for understanding patterns of daily behavior [32, $59,60]$. It is worth acknowledging however, the benefit of further extending the wear period to investigate adherence within or across NDD cohorts when the wear duration is lengthened. Extended wear could increase the likelihood of capturing infrequent events or changes in behavior that occur over a longer term (e.g., seasonal effects, medication titration) and minimize the impact of modifications in behavior that could occur when participants are initially instrumented with sensors. In the current study, increases in non-wear for a few participants later in the study period may suggest that such extended wear could be problematic for some individuals. A study by Silva de Lima et al. [31] evaluated long-term adherence to a wearables protocol in a cohort of persons living with PD. When assessed across a 13-week period, investigators reported an attrition rate of $23 \%$ and a lower median compliance rate $(68 \%)$ than what was found in the present study ( $98 \%$ sensor wear), with low ratings of system usability differentiating participants who were most from least compliant [31]. As such, future work should focus on optimizing extended multi-sensor wear by reducing barriers where possible.

Examining patterns and differences in device removal at the individual level is distinct from previous work that has largely focused on group-level metrics of adherence [26, 30]. Despite a considerable range in level of disability (as screened by $\mathrm{mRS}$ ) and cognitive functioning (as screened 
by MoCA), multi-sensor wear time was high across all participants. For four of the five participants with the lowest adherence rates (ranging from 8 to $30 \%$ non-wear), gross measures of cognitive and physical status did not differentiate these participants from others in the group. This finding aligns with other studies that assessed the feasibility of wearable sensors and reported that clinical characteristics, such as disease severity, loss of independence, or cognitive impairment, did not significantly influence compliance at the group level $[31,56]$. In addition to total non-wear time, examining the duration and frequency of non-wear bouts at the individual level provided novel insights into the daily pattern of device removal within and between participants. Specifically, extended periods of removal (i.e. greater than $5 \mathrm{~h}$ ) were infrequent and not confined to a particular cohort. In fact, these extended periods occurred only in the five participants with the highest amounts of non-wear, and these participants represented four of the five study cohorts. For the remaining participants, non-wear occurred in short bouts and often at consistent times across days, suggesting that participants did not experience issues that made them "abandon" the wear protocol for lengthy periods of time.

Feedback during the de-brief interviews with participants and study partners was positive overall, with comfort, ease of use, appearance, and ability to perform ADLs without interference emerging as important factors related to the usability and acceptability of the approach. Some participants and study partners also noted a desire to receive sensor-derived feedback that may provide insight into disease or health-related behaviors. The generally positive response suggests that this model could be implemented with minimal interruption to usual behavior. However, some participants and study partners did identify barriers and concerns related to the set up and although these views did not translate into poor adherence, there is an important need to address this feedback to optimize the approach. Specifically, participant comments about skin redness or itchiness and/or mild discomfort related to the ECG adhesive highlight the need to consider age or disease-related changes to skin integrity and suggest that a wear schedule inclusive of breaks may be warranted (e.g., three days on, one day off). Decisions regarding wear schedule must also consider the timescale needed to capture measures of interest (e.g., duration of continuous wear needed to capture day-night reversals). For participants who identified challenges with donning the devices independently or expressed concerns about device attachment, consideration should be given to providing a range of attachment methods that cater to participant preference, specific devices, and wear location [33]. Although the current study adopted the use of medical-grade wrap to allow for comfortable long-term wear of ankle devices, concern about having to remove devices for water-based ADLs may be addressed by using waterproof technology or coverings.
Last, the preference expressed by both participants and study partners for thinner, smaller devices that may be less noticeable supports the need to continue to evaluate new technology options and form factors that emerge with potential promise for CVD and NDD applications. As it relates to this matter of wearability, advances in technology should strive to accommodate different wear locations with a view to optimize data capture for the physiological systems, behaviors, or symptoms of disease that the devices are intended to measure. Practically, the suggestions provided in this study to address barriers to device wear may help to balance participant preference for wear location with the location that provides the most meaningful data. Overall, the concerns identified by participants and study partners highlight the importance of engaging end users in the development of extended wear protocols to optimize acceptability and adherence to device wearing in free-living contexts [61].

Importantly, it is possible the high acceptance and adherence reported within this study was influenced by the intentional strategies built into the protocol to achieve the following: (1) limit participant engagement with devices (the devices required no interaction beyond attachment and removal), and (2) offset device-related challenges experienced by participants by including a study partner and providing access to a research coordinator. Previous studies have reported that adherence depended on the complexity of the technology being used [62] and the degree of user engagement required $[27,56]$. No participants required research coordinator support to engage with the devices; however, one study partner did receive additional support from the study team when a participant became agitated by the devices. This instance reinforces the importance of embedding support mechanisms [31] and the need to consider the role of the care partner, as well as the potential impact of caregiver burden, in a remote monitoring model $[33,56]$. Such supports must be considered for their scalability in the context of large clinical trials. Further, other study- or participant-specific features such as physiciandriven recruitment, the inclusion of personalized feedback, and participants' education level may have motivated participation and positively impacted acceptance and adherence.

In conclusion, this work has shown that a continuous, multi-sensor remote health monitoring approach is feasible and well tolerated by a cohort of persons living with CVD, AD/MCI, FTD, PD, or ALS. Ongoing work continues to focus on establishing the essential subset of sensors (type and wear location) and supporting data analytics to effectively describe behavioral, physiological and clinical characteristics relevant to persons with CVD or NDD, their care partner, and their clinicians [6, 63]. Remote monitoring technologies must also support the wearability and tolerance for use of more than a single device and provide access to unprocessed raw data, which will enable development 
of new analytics to optimize outputs from a multi-sensor approach. The results of the present study provide important consideration for the launch of larger remote monitoring studies across or within these populations, with future implications for clinical care and the ability to capture meaningful health outcomes in more ecologically valid settings.

Acknowledgements The authors thank ReMiNDD participants and study partners, as well as ONDRI investigators and the ONDRI project management team for their input and administrative support of this project.

Author contributions AR, RHS, PMM, SEB, MM, LZ, MB, AL, DM, SCS, WEM and KVO contributed to the study conception and design. ET, YD, RHS, AA, SEB, MM, and LZ assisted with participant recruitment. Study coordinator training, material preparation, and data collection were performed by ET, YD, AR, PMM, KSW, WEM and KVO. Data management, curation and analysis were performed by FEG, ET, AV, PMM, KSW, VT, KBB, BC, DB, VC, DK, KMS, BT, WEM and KVO. The first draft of the manuscript was written by FEG, ET and $\mathrm{KVO}$ and all authors commented on previous versions of the manuscript. All authors read and approved the final manuscript.

Funding This research was conducted with the support of the Ontario Brain Institute, an independent non-profit corporation, funded partially by the Ontario government. The opinions, results, and conclusions are those of the authors and no endorsement by the Ontario Brain Institute is intended or should be inferred.

Availability of data and material ONDRI data-including but not limited to the data in this article-will be accessible through the Ontario Brain Institute (https://braininstitute.ca/) to qualified researchers. Please see the Ontario Brain Institute website for information on when the data will be released and how to access the data: https://braininsti tute.ca/.

Code availability Not applicable.

\section{Declarations}

Conflicts of interest On behalf of all authors, the corresponding author states that there is no conflict of interest.

Ethical approval This study was performed in line with the principles of the Declaration of Helsinki. Approval was granted by the Sunnybrook Health Sciences Centre research ethics board (March 14, 2019, No.: 1832).

Consent to participate Informed consent was obtained from all individual participants and study partners included in the study.

Consent for publication The authors affirm that human research participants provided informed consent for publication of their data.

\section{References}

1. Morgan C, Rolinski M, McNaney R et al (2020) Systematic review looking at the use of technology to measure free-living symptom and activity outcomes in Parkinson's disease in the home or a home-like environment. J Parkinsons Dis 10:429-454. https://doi. org/10.3233/JPD-191781

2. Bonato $P$ (2010) Wearable sensors and systems: from enabling technology to clinical applications. IEEE Eng Med Biol Mag 29:25-36. https://doi.org/10.1109/MEMB.2010.936554

3. Del Din S, Godfrey A, Mazzà C et al (2016) Free-living monitoring of Parkinson's disease: lessons from the field. Mov Disord 31:1293-1313. https://doi.org/10.1002/mds.26718

4. Mancini M, Shah VV, Stuart S et al (2021) Measuring freezing of gait during daily-life: an open-source, wearable sensors approach. J Neuroeng Rehabil 18:1-13. https://doi.org/10.1186/ s12984-020-00774-3

5. Papapetropoulos S, Mitsi G, Espay AJ (2015) Digital health revolution: is it time for affordable remote monitoring for Parkinson's disease? Front Neurol 6:1-3. https://doi.org/10.3389/fneur.2015. 00034

6. Espay AJ, Bonato P, Nahab F et al (2016) Technology in Parkinson disease: challenges and opportunities. Mov Disord 31:12721282. https://doi.org/10.1002/mds.26642

7. Patel S, Park H, Bonato P et al (2012) A review of wearable sensors and systems with application in rehabilitation. J Neuroengineering Rehabil. https://doi.org/10.1186/1743-0003-9-21

8. Ryan DJ, Wullems JA, Stebbings GK et al (2018) Reliability and validity of the international physical activity questionnaire compared to calibrated accelerometer cut-off points in the quantification of sedentary behavior and physical activity in older adults. PLoS ONE 13:e0195712. https://doi.org/10.1371/journal.pone. 0195712

9. Chaudhuri S, Thompson H, Demiris G (2014) Fall detection devices and their use with older adults: a systematic review. J Geriatr Phy Ther 37:178-196. https://doi.org/10.1519/JPT.0b013 e3182abe779

10. Maetzler W, Domingos J, Srulijes K et al (2013) Quantitative wearable sensors for objective assessment of Parkinson's disease. Mov Disord 28:1628-1637. https://doi.org/10.1002/mds.25628

11. Adams JL, Myers TL, Waddell EM et al (2020) Telemedicine: a valuable tool in neurodegenerative diseases. Curr Geriatr Reports 9:72-81. https://doi.org/10.1007/s13670-020-00311-z

12. Elbeddini A, To A, Tayefehchamani Y, Wen C (2020) Potential impact and challenges associated with Parkinson's disease patient care amidst the COVID-19 global pandemic. J Clin Mov Disord. https://doi.org/10.1186/s40734-020-00089-4

13. Evans L, Mohamed B, Thomas EC (2020) Using telemedicine and wearable technology to establish a virtual clinic for people with Parkinson's disease. BMJ open Qual 9:e001000. https://doi.org/ 10.1136/bmjoq-2020-001000

14. Erb MK, Karlin DR, Ho BK et al (2020) mHealth and wearable technology should replace motor diaries to track motor fluctuations in Parkinson's disease. npj Digit Med 3:1-20. https://doi.org/ 10.1038/s41746-019-0214-x

15. Vizcarra JA, Sánchez-Ferro Á, Maetzler W et al (2019) The Parkinson's disease e-diary: developing a clinical and research tool for the digital age. Mov Disord 34:676-681. https://doi.org/10. $1002 / \mathrm{mds} .27673$

16. Chiauzzi E, Rodarte C, DasMahapatra P (2015) Patient-centered activity monitoring in the self-management of chronic health conditions. BMC Med 13:1-6. https://doi.org/10.1186/ s12916-015-0319-2

17. Espay AJ, Hausdorff JM, Sánchez-Ferro Á et al (2019) A roadmap for implementation of patient-centered digital outcome measures in Parkinson's disease obtained using mobile health technologies. Mov Disord 34:657-663. https://doi.org/10.1002/mds.27671

18. Sommer R, Yu L, Schneider JA et al (2021) Disrupted rest-activity rhythms and cerebral small vessel disease pathology in older adults. Stroke. https://doi.org/10.1161/strokeaha.120.030870 
19. McCurry SM, Pike KC, Vitiello MV et al (2011) Increasing walking and bright light exposure to improve sleep in communitydwelling persons with Alzheimer's disease: Results of a randomized, controlled trial. J Am Geriatr Soc 59:1393-1402. https:// doi.org/10.1111/j.1532-5415.2011.03519.x

20. Memon AA, Coleman JJ, Amara AW (2020) Effects of exercise on sleep in neurodegenerative disease. Neurobiol Dis 140:104859. https://doi.org/10.1016/j.nbd.2020.104859

21. Storm FA, Buckley CJ, Mazzà C (2016) Gait event detection in laboratory and real life settings: accuracy of ankle and waist sensor based methods. Gait Posture 50:42-46. https://doi.org/10. 1016/j.gaitpost.2016.08.012

22. Camps J, Samà A, Martín M et al (2018) Deep learning for freezing of gait detection in Parkinson's disease patients in their homes using a waist-worn inertial measurement unit. Knowledge-Based Syst 139:119-131. https://doi.org/10.1016/j.knosys.2017.10.017

23. Boe AJ, McGee Koch LL, O'Brien MK et al (2019) Automating sleep stage classification using wireless, wearable sensors. npj Digit Med 2:131. https://doi.org/10.1038/s41746-019-0210-1

24. Strath SJ, Bassett DR, Swartz AM, Thompson DL (2001) Simultaneous heart rate-motion sensor technique to estimate energy expenditure. Med Sci Sport Exerc 33:2118-2123. https://doi.org/ 10.1097/00005768-200112000-00022

25. Botros A, Schütz N, Camenzind M et al (2019) Long-term homemonitoring sensor technology in patients with parkinson's disease-acceptance and adherence. Sensors (Switzerland) 19:5169. https://doi.org/10.3390/s19235169

26. Fisher JM, Hammerla NY, Rochester L et al (2016) Body-worn sensors in Parkinson's disease: evaluating their acceptability to patients. Telemed e-Health 22:63-69. https://doi.org/10.1089/tmj. 2015.0026

27. Ancona S, Faraci FD, Khatab E et al (2021) Wearables in the home-based assessment of abnormal movements in Parkinson's disease: a systematic review of the literature. J Neurol. https://doi. org/10.1007/s00415-020-10350-3

28. Adams JL, Dinesh K, Xiong M et al (2017) Multiple wearable sensors in Parkinson and Huntington disease individuals: a pilot study in clinic and at home. Digit Biomarkers 1:52-63. https:// doi.org/10.1159/000479018

29. Bayés À, Samá A, Prats A et al (2018) A "HOLTER" for Parkinson's disease: validation of the ability to detect on-off states using the REMPARK system. Gait Posture 59:1-6. https://doi.org/10. 1016/j.gaitpost.2017.09.031

30. Boroojerdi B, Ghaffari R, Mahadevan N et al (2019) Clinical feasibility of a wearable, conformable sensor patch to monitor motor symptoms in Parkinson's disease. Park Relat Disord 61:70-76. https://doi.org/10.1016/j.parkreldis.2018.11.024

31. Silva de Lima AL, Hahn T, Evers LJW et al (2017) Feasibility of large-scale deployment of multiple wearable sensors in Parkinson's disease. PLoS ONE 12:e0189161. https://doi.org/10.1371/journal. pone.0189161

32. Camargos EF, Louzada FM, Nóbrega OT (2013) Wrist actigraphy for measuring sleep in intervention studies with Alzheimer's disease patients: Application, usefulness, and challenges. Sleep Med Rev 17:475-488. https://doi.org/10.1016/j.smrv.2013.01.006

33. Thordardottir B, Malmgren Fänge A, Lethin C et al (2019) Acceptance and use of innovative assistive technologies among people with cognitive impairment and their caregivers: a systematic review. Biomed Res Int 2019:9196729. https://doi.org/10.1155/2019/91967 29

34. Farhan SMK, Bartha R, Black SE et al (2017) The Ontario Neurodegenerative Disease Research Initiative (ONDRI). Can J Neurol Sci 44:196-202. https://doi.org/10.1017/cjn.2016.415

35. Marian V, Blumenfeld HK, Kaushanskaya M (2007) The Language Experience and Proficiency Questionnaire (LEAP-Q): assessing language profiles in bilinguals and multilinguals. J Speech, Lang Hear Res 50:940-967. https://doi.org/10.1044/1092-4388(2007/067)

36. Buysse DJ, Reynolds CF, Monk TH et al (1989) The Pittsburgh Sleep Quality Index: a new instrument for psychiatric practice and research. Psychiatry Res 28:193-213. https://doi.org/10.1016/01651781(89)90047-4

37. Spitzer RL, Kroenke K, Williams JBW, Löwe B (2006) A brief measure for assessing generalized anxiety disorder: The GAD-7. Arch Intern Med 166:1092-1097. https://doi.org/10.1001/archinte. 166.10.1092

38. Rush AJ, Trivedi MH, Ibrahim HM et al (2003) The 16-item Quick Inventory of Depressive Symptomatology (QIDS), clinician rating (QIDS-C), and self-report (QIDS-SR): A psychometric evaluation in patients with chronic major depression. Biol Psychiatry 54:573-583. https://doi.org/10.1016/S0006-3223(02)01866-8

39. Nasreddine ZS, Phillips NA, Bédirian V et al (2005) The Montreal Cognitive Assessment, MoCA: a brief screening tool for mild cognitive impairment. J Am Geriatr Soc 53:695-699. https://doi.org/10. 1111/j.1532-5415.2005.53221.x

40. van Swieten J, Koudstaal PJ, Visser MC et al (1988) Interobserver agreement for the assessment of handicap in stroke patients. Stroke 19:604-607. https://doi.org/10.1161/01.str.19.5.604

41. Harris PA, Taylor R, Thielke R et al (2009) Research electronic data capture (REDCap)-A metadata-driven methodology and workflow process for providing translational research informatics support. J Biomed Inform 42:377-381. https://doi.org/10.1016/j.jbi.2008.08. 010

42. Harris PA, Taylor R, Minor BL et al (2019) The REDCap consortium: building an international community of software platform partners. J Biomed Inform 95:103208. https://doi.org/10.1016/j.jbi. 2019.103208

43. Baltadjieva R, Giladi N, Gruendlinger L et al (2006) Marked alterations in the gait timing and rhythmicity of patients with de novo Parkinson's disease. Eur J Neurosci 24:1815-1820. https://doi.org/ 10.1111/j.1460-9568.2006.05033.x

44. Ravits J, Paul P, Jorg C (2007) Focality of upper and lower motor neuron degeneration at the clinical onset of ALS. Neurology 68:1571-1575. https://doi.org/10.1212/01.wnl.0000260965.20021. 47

45. Lam E, Aratia S, Wang J, Tung J (2020) Measuring heart rate variability in free-living conditions using consumer-grade photoplethysmography: validation study. JMIR Biomed Eng 5:e17355. https:// doi.org/10.2196/17355

46. Kourtis LC, Regele OB, Wright JM, Jones GB (2019) Digital biomarkers for Alzheimer's disease: the mobile/wearable devices opportunity. npj Digit Med. https://doi.org/10.1038/s41746-019-0084-2

47. Perez M, Jin W, Le D et al (2018) Classification of Huntington disease using acoustic and lexical features. Interspeech 2018:1898-1902

48. Romana A, Bandon J, Carlozzi N et al (2020) Classification of manifest Huntington disease using vowel distortion measures. Interspeech 2020:4966-4970

49. Sandelowski M (2000) Whatever happened to qualitative description? Res Nurs Heal 23:334-340. https://doi.org/10.1002/1098240x(200008)23:4\%3c334::aid-nur9\%3e3.0.co;2-g

50. Van Ooteghem K, Mansfield A, Inness EL et al (2020) Integrating technology into clinical practice for the assessment of balance and mobility: perspectives of exercise professionals practicing in retirement and long-term care. Arch Rehabil Res Clin Transl 2:100041. https://doi.org/10.1016/j.arrct.2020.100041

51. Sunderland KM, Beaton D, Fraser J et al (2019) The utility of multivariate outlier detection techniques for data quality evaluation in large studies: an application within the ONDRI project. BMC Med Res Methodol. https://doi.org/10.1186/s12874-019-0737-5

52. Syed S, Morseth B, Hopstock LA, Horsch A (2020) Evaluating the performance of raw and epoch non-wear algorithms using multiple 
accelerometers and electrocardiogram recordings. Sci Rep 10:5866. https://doi.org/10.1038/s41598-020-62821-2

53. Brunner E, Domhof S, Langer F (2002) Nonparametric analysis of longitudinal data in factorial experiments. J Wiley

54. Sunderland KM, Beaton D, Arnott SR et al (2020) The Ontario Neurodegenerative Disease Research Initiative (ONDRI). medRXiv. https://doi.org/10.1101/2020.07.30.20165456

55. Heijmans M, Habets JGV, Herff C et al (2019) Monitoring Parkinson's disease symptoms during daily life: a feasibility study. npj Park Dis 5:1-6. https://doi.org/10.1038/s41531-019-0093-5

56. Gatsios D, Antonini A, Gentile G et al (2020) Feasibility and utility of mhealth for the remote monitoring of parkinson disease: ancillary study of the PD_manager randomized controlled trial. JMIR mHealth uHealth 8:e16414. https://doi.org/10.2196/16414

57. Serfaty M, Kennell-Webb S, Warner J et al (2002) Double blind randodmised placebo controlled trial of low dose melatonin for sleep disorders in dementia. Int J Geriatr Psychiatry 17:1120-1127. https://doi.org/10.1002/gps.760

58. Skender S, Ose J, Chang-Claude J et al (2016) Accelerometry and physical activity questionnaires - a systematic review. BMC Public Health 16:515. https://doi.org/10.1186/s12889-016-3172-0
59. Hart T, Swartz A, Cashin S, Strath S (2011) How many days of monitoring predict physical activity and sedentary behavior in older adults? Int J Behav Nutr Phys Act 8:62. https://doi.org/10.1186/ 1479-5868-8-62

60. Lim ASP, Yu L, Costa MD et al (2011) Quantification of the fragmentation of rest-activity patterns in elderly individuals using a state transition analysis. Sleep 34:1569-1581. https://doi.org/10.5665/ sleep. 1400

61. Piau A, Wild K, Mattek N, Kaye J (2019) Current state of digital biomarker technologies for real-life, home-based monitoring of cognitive function for mild cognitive impairment to mild Alzheimer disease and implications for clinical care: systematic review. J Med Internet Res 21:e12785. https://doi.org/10.2196/12785

62. Cahill S, Begley E, Faulkner JP, Hagen I (2007) "It gives me a sense of independence" - Findings from Ireland on the use and usefulness of assistive technology for people with dementia. Technol Disabil 19:133-142. https://doi.org/10.3233/tad-2007-192-310

63. Brognara L, Palumbo P, Grimm B, Palmerini L (2019) Assessing gait in Parkinson's disease using wearable motion sensors: a systematic review. Diseases 7:18. https://doi.org/10.3390/diseases7010018 